\title{
The Hunt for the First Supernovae: The Source Density and Observability of Pair-Instability Supernovae from the First Stars
}

\section{Jacob A. Hummel*, Andreas H. Pawlik, Miloš Milosavljević, and Volker Bromm}

Department of Astronomy and Texas Cosmology Center, The University of Texas at Austin

E-mail: jhummel@astro.as.utexas.edu

\begin{abstract}
Some of the first stars are expected to have died as extremely energetic pair-instability supernovae (PISNe). With energies approaching $10^{53} \mathrm{ergs}$, these supernovae are expected to be within the detection limits of the upcoming James Webb Space Telescope (JWST), allowing us to constrain the properties of these objects for the first time. We estimate the source density of PISNe using a semi-analytic Press-Schechter based approach informed by cosmological simulations including feedback from star formation. We find that the main obstacle to detecting PISNe is their scarcity, not their brightness; exposures longer than a few times $10^{4} \mathrm{~s}$ will do little to increase the number of PISNe found. Given this we suggest a mosaic style search strategy for detecting PISNe from the first stars. Even fairly high redshift PISNe are sufficiently bright to be found with moderately deep exposures. However, a large number of pointings will be required to ensure a detection due to their scarcity. For an observing program totalling $10^{6} \mathrm{~s}$, the probability of a detection is maximized by dividing the campaign into $\sim 150$ individual fields with a $\sim 5000$ s exposure in each.
\end{abstract}

Frank N. Bash Symposium New Horizons In Astronomy,

October 9-11, 2011

Austin Texas

* Speaker. 


\section{Introduction}

Following the launch of the upcoming James Webb Space Telescope (JWST) we will be able to probe the epoch of the first stars with unprecedented detail [1]. These so-called Population III (Pop III) stars formed in $10^{5}-10^{6} M_{\odot}$ dark matter 'minihalos' at high redshifts and were predominantly very massive (e.g., $[2,3]$ ). Recent work has revised this picture somewhat. Significant fragmentation likely occurred in the primordial gas clouds from which the first stars were born, lowering their characteristic masses from $100 M_{\odot}$ to nearer $50 M_{\odot}$, with a much broader initial mass function and significant rotation expected as well $[4,5,6]$. While the first stars themselves are unlikely to be visible [7], it is possible that some of the first stars died as pair-instability supernovae (PISNe). Non-rotating stars in the range $140-260 M_{\odot}$ are expected to die as PISNe, undergoing a pair-production instability following core oxygen burning to explode completely. These extremely energetic explosions - approaching a total energy of $10^{53}$ ergs - will be within the detection limits of the JWST $[8,9]$. It has recently been found that the inclusion of rotation can significantly alter the initial mass required for a star to meet this fate, lowering the minimum mass required to encounter the pair-production instability to as little as $65 M_{\odot}$ for the most rapidly rotating models [10]. While the basic properties of PISNe and the effect they have on their environment has been well studied, the source density of these events has yet to be well constrained. Our work complements previous investigations of the PISN rate (e.g., [9, 11, 12, 13, 14]) by including an extended discussion of the effects of feedback from star formation on the PISN rate, considering LymanWerner (LW) feedback and chemical feedback. We describe here our semi-analytic model for the PISN rate and assess the ability of the JWST to detect PISNe from the first stars. For further details see Hummel et al. [15], on which this work is based.

\section{The PISN Rate}

It is reasonable to assume that at most one PISN will occur per minihalo. PISNe are produced only by very massive stars. After the first star forms, photoionization quickly suppresses the density of the remaining gas, effectively halting star formation in the minihalo. The energy released by the first PISN disperses the gas in the halo, delaying star formation until the gas is able to recondense into more massive halos. While star formation resumes at this point, the gas in these systems will likely be enriched beyond the critical metallicity for Pop II star formation. As a result, the stars that form will no longer be large enough to reliably produce PISNe. Assuming the time required for the progenitor star to form, live and die is negligible, the formation rate of minihalos serves as a robust upper limit for the PISN rate. To quantify this, we assume exactly one PISN per minihalo [15], forming as soon as the halo exceeds the critical mass required for star formation. We then use the analytic Press-Schechter (PS) formalism for structure formation [16] to calculate the number density $n_{\mathrm{PS}}$ of critical mass minihalos at redshift $z$, estimating their formation rate $\dot{n}_{+}(z)$ using the expression derived by [17]. The resulting rate can be seen in Figure $1 \mathrm{~b}$.

Stellar feedback will suppress Pop III star formation in some minihalos and completely halt it in others, reducing the PISN rate. The feedback mechanisms responsible for this include the build-up of a background of $\mathrm{H}_{2}$ dissociating LW photons and chemical feedback polluting the gas with metals, halting Pop III star formation. These feedback mechanisms can be represented by 
distinct efficiency factors $\eta(z)$, such that the true PISN rate, $\dot{n}_{\mathrm{PISN}}$, will be given by $\dot{n}_{\mathrm{PISN}}(z)=$ $\eta_{\text {chem }}(z) \eta_{\text {LW }}(z) \dot{n}_{+}(z)$. To asses the impact of LW feedback we employ a set of two cosmological simulations. The first simulation we employ is similar to simulation Z4 presented in [7]. This simulation follows the assembly of a galaxy in a halo reaching a mass of $\sim 10^{9} M_{\odot}$ at $z=10$, including star formation but ignoring the associated feedback. The second simulation differs only in the inclusion of LW feedback. Determining the critical mass for star formation with and without LW feedback, the efficiency of LW feedback, $\eta_{\mathrm{LW}}$, can then be expressed as the ratio of the formation rate of minihalos at the critical mass with LW feedback to that without. The resulting efficiency factor $\eta_{\mathrm{LW}}$ is shown in Figure 1a, and the LW modulated PISN rate in Figure 1b. Once the critical mass reaches the atomic cooling threshold at $z \sim 12$, LW feedback is no longer efficient. The

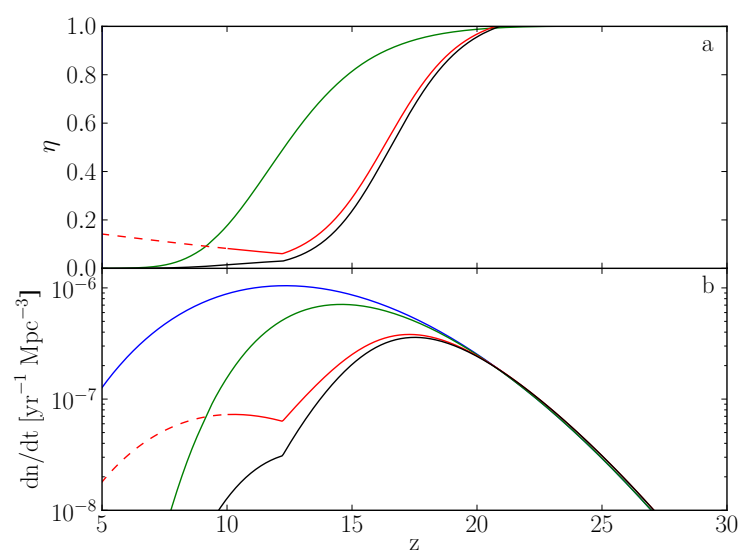

Figure 1: a) Evolution of the feedback efficiency factors $\eta$ as a function of redshift. $\eta_{\text {chem }}$ is shown in green and $\eta_{\mathrm{Lw}}$ in red; $\eta_{\text {tot }} \equiv \eta_{\text {chem }} \eta_{\mathrm{LW}}$ is shown in black. The feature at $z \sim 12$ is due to the critical mass reaching the atomic cooling threshold. b) The PISN rate in the upper limit of no feedback (blue), with chemical feedback (green), LW feedback (red) and the final predicted PISN rate (black).

process of chemical enrichment is another crucial factor for determining the PISN rate. Gas that has been enriched beyond a critical metallicity of $Z_{\text {crit }} \sim 10^{-4} Z_{\odot}$ will no longer form Pop III stars [18], and hence no PISNe. The effects of chemical feedback can thus be evaluated by computing the fraction of halos forming from pristine gas at a given redshift. In modeling $\eta_{\text {chem }}$, we use the results of Furlanetto \& Loeb [19]. Their semi-analytic treatment of SN winds yields a probability function $P_{\text {pristine }}(z)$ that the gas in a newly formed halo is pristine. We identify this quantity as the fraction of newly collapsed halos polluted with metals, $\eta_{\text {chem }}$. Given the recent detection of pristine gas at $z=3$ by [20], we choose the weakest feedback scenario presented. The selected $\eta_{\text {chem }}$ can be seen in Figure 1a, and the resulting PISN rate in Figure 1b.

\section{JWST Observability}

To determine the observability of PISNe at high redshifts we consider a representative case, that of the $250 M_{\odot}$ PISN model presented in [21]. Given the large mass ejected, the ejecta will remain optically thick until late times, so we make the reasonable assumption that the PISN emits 
as a blackbody for the majority of its visible lifetime. The resulting lightcurves as they would appear at various redshifts are shown in the left panel of Figure 2. For further details see [15].
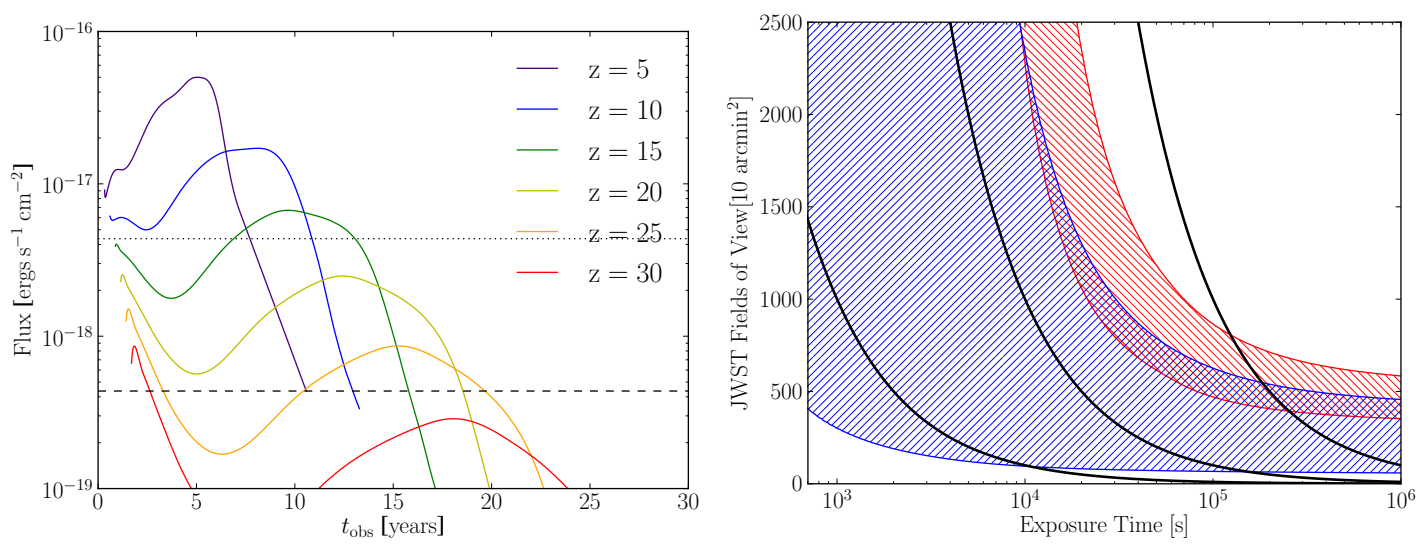

Figure 2: Left: Lightcurves for the PISN model as it would be observed by JWST's F444W NIRCam filter at $z=$ $5,10,15,20,25$ and 30. The flux limits for a $10^{6} \mathrm{~s}$ (dashed line) and $10^{4} \mathrm{~s}$ (dotted line) are shown for reference. Right: The observability of PISNe using the JWST's NIRcam F444W filter. Shown are the lower and upper limits for the number of JWST FoVs required to detect 10 sources as a function of exposure time. The blue range denotes the limits for PISNe from all redshifts, and the red for PISNe from $z>15$. The lower limits of the ranges correspond to the no-feedback upper limit to the PISN rate and the upper limits to the rate derived with feedback. From bottom to top, the black lines represent the number of pointings possible in a total of $10^{6} \mathrm{~s}, 10^{7} \mathrm{~s}$ and $10^{8} \mathrm{~s}$.

\section{Discussion and Conclusions}

We conclude that the limiting factor in detecting PISNe will be the scarcity of sources rather than their faintness, in agreement with the results of [12]. PISNe should be readily detectable out to $z \sim 20$, but beyond a moderate exposure time of a few times $10^{4} \mathrm{~s}$ their observability is controlled almost completely by the source density-approximately one PISN per 5 JWST fields of view (FoVs) above $z=10$, and dropping off quickly beyond $z \sim 15$. This is clear from the right panel of Figure 2, where we have shown the number of JWST FoVs required to detect $10 \mathrm{PISNe}$ (in blue) as a function of exposure time. Even for only high-redshift sources $(z>15$, in red) the dependence on exposure time is minimal, being controlled by the lack of sources. While the detection of a PISN from a 'first' star at very high redshifts would be exciting and is in fact possible given the detection limits of the JWST, the scarcity of sources at these redshifts means that such a detection would be contingent on serendipity. The detection of a PISN at lower redshifts however appears to be within the realm of possibility. In this case, the strategy with the highest likelihood of detection will be a mosaic survey consisting of approximately 150 pointings to a depth of $\sim 5000 \mathrm{~s}$.

\section{Acknowledgments}

V.B. and M.M. acknowledge support from NSF grants AST-0708795 and AST-1009928 and NASA ATFP grant NNX09AJ33G. V.B. thanks the Max-Planck-Institut für Astrophysik for its hospitality during part of the work on this paper. The simulations were carried out at the Texas Advanced Computing Center (TACC). 


\section{References}

[1] Gardner, J. P., et al., The James Webb Space Telescope, 2006, Space Sci. Rev. 123, 485

[2] Bromm, V., Coppi, P. S. \& Larson, R. B. Forming the first stars in the universe: The fragmentation of primordial gas, 1999, ApJL 527, L5

[3] Abel, T., Bryan, G. L. \& Norman, M. L. The formation of the first star in the Universe, 2002, Science 295, 93

[4] Stacy, A., Greif, T. H. \& Bromm, V. The first stars: formation of binaries and small multiple systems, 2010, MNRAS 403, 45

[5] Clark, P. C. et al. The formation and fragmentation of disks around primordial protostars, 2011, Science 331,1040

[6] Greif, T. H. et al. Simulations on a Moving Mesh: the Clustered Formation of Population III Protostars, 2011, ApJ 737, 75

[7] Pawlik, A. H., Milosavljević, M. \& Bromm, V. The First Galaxies: Assembly of Disks and Prospects for Direct Detection, 2011, ApJ 731, 54

[8] Heger, A., Fryer, C. L., Woosley, S. E., Langer, N. \& Hartmann, D. H. How massive single stars end their life, 2003, ApJ 591, 288

[9] Scannapieco, E., Madau, P., Woosley, S. E., Heger, A. \& Ferrara, A. The Detectability of Pair-Production Supernovae at $z \lesssim 6,2005$, ApJ 633, 1031

[10] Chatzopoulos, E. \& Wheeler, J. C. Effects of Rotation on the Minimum Mass of Primordial Progenitors of Pair Instability Supernovae, 2012, ApJ, in press (arXiv:1201.1328v2)

[11] Mackey, J., Bromm, V. \& Hernquist, L. Three epochs of star formation in the high-redshift Universe, 2003, ApJ 586, 1

[12] Weinmann, S. M. \& Lilly, S. J. The Number and Observability of Population III Supernovae at High Redshifts, 2005, ApJ 624, 526

[13] Wise, J. H. \& Abel, T. The Number of Supernovae from Primordial Stars in the Universe, 2005, ApJ 629, 615

[14] Trenti, M., Stiavelli, M. \& Michael Shull, J. Metal-Free Gas Supply at the Edge of Reionization: Late-Epoch Population III Star Formation, 2009, ApJ 700, 1672

[15] Hummel, J., Pawlik, A., Milosavljevic, M. \& Bromm, V. The Source Density and Observability of Pair-Instability Supernovae from the First Stars, 2011, ApJ, submitted (arXiv:1112.5207)

[16] Press, W. H. \& Schechter, P. Formation of Galaxies and Clusters of Galaxies by Self-Similar Gravitational Condensation, 1974, ApJ 187, 425

[17] Sasaki, S. Formation Rate of Bound Objects in the Hierarchical Clustering Model, 1994, PASJ 46, 427

[18] Schneider, R., Ferrara, A., Natarajan, P. \& Omukai, K. First Stars, Very Massive Black Holes, and Metals, 2002, ApJ 571, 30

[19] Furlanetto, S. R. \& Loeb, A. Is Double Reionization Physically Plausible? 2005, ApJ 634, 1

[20] Fumagalli, M., O’Meara, J. M. \& Prochaska, J. X. Detection of Pristine Gas Two Billion Years After the Big Bang, 2011, Science 334, 1245

[21] Kasen, D., Woosley, S. E. \& Heger, A. Pair Instability Supernovae: Light Curves, Spectra, and Shock Breakout, 2011, ApJ 734, 102 